\title{
On Beltrami fields with nonconstant proportionality factor on the plane
}

\author{
Vladislav V. Kravchenko ${ }^{1}$, Héctor Oviedo ${ }^{2}$ \\ ${ }^{1}$ Department of Mathematics, CINVESTAV del IPN, Unidad Queretaro, \\ Libramiento Norponiente No. 2000, Fracc. Real de Juriquilla, Queretaro, \\ Qro. C.P. 76230 MEXICO e-mail: vkravchenko@qro.cinvestav.mx* \\ ${ }^{2}$ SEPI, ESIME Zacatenco, Instituto Politécnico Nacional, Av. IPN S/N, \\ C.P. 07738, D.F. MEXICO $^{\dagger}$
}

November 2, 2018

\begin{abstract}
We consider the equation

$$
\operatorname{rot} \vec{B}+\alpha \vec{B}=0
$$

on the plane with $\alpha$ being a real-valued function and show that it can be reduced to a Vekua equation of a special form. In the case when $\alpha$ depends on one Cartesian variable a complete system of exact solutions of the Vekua equation and hence of equation (1) is constructed based on L. Bers' theory of pseudoanalytic formal powers.
\end{abstract}

\section{Introduction}

Solutions of the equation

$$
\operatorname{rot} \vec{B}+\alpha \vec{B}=0
$$

\footnotetext{
*Research was supported by CONACYT, Mexico via the research project 50424 .

†During the preparation of this work the second-named author was supported by CONACYT on a postdoctoral stay at the Department of Mathematics, CINVESTAV del IPN, Unidad Querétaro
} 
where $\alpha$ is a scalar function of space coordinates are known as Beltrami fields and are of fundamental importance in different branches of modern physics (see, e.g., [22], [18, [7], 21], [1], 9], [8], 11]). For simplicity, in this work we consider the real-valued proportionality factor $\alpha$ and real-valued solutions of (2), though the presented approach is applicable in a complexvalued situation as well with a considerable complication of mathematical techniques involved (instead of complex Vekua equations their bicomplex generalizations should be considered [5], [14]). We consider equation (2) on a plane of the variables $x$ and $y$, that is $\alpha$ and $\vec{B}$ are functions of two Cartesian variables only. In this case as we show in section 3 equation (2) reduces to the equation

$$
\operatorname{div}\left(\frac{1}{\alpha} \nabla u\right)+\alpha u=0 .
$$

This second-order equation can be reduced (see [14]) to a corresponding Vekua equation (describing generalized analytic functions) of a special form. This reduction under quite general conditions allows us to construct a complete system of exact solutions of (3) explicitly (see [13] and [15]). For the reduction of (3) to a Vekua equation it is sufficient to find a particular solution of (3). In the present work (section 4) we show that in a very important for applications case of $\alpha$ being a function of one Cartesian variable a particular solution of (3) is always available in a simple explicit form. This situation corresponds to models describing waves propagating in stratified media (see, e.g., [16]). As a result in this case we are able to construct a complete system of solutions explicitly which for many purposes means a general solution. We give an example of such construction.

We show in this work that when $\alpha=\alpha(y)$ (of course in a similar way the case $\alpha=\alpha(x)$ can be considered) equation (3) and hence equation (2) reduce to the Vekua equation of the following form

$$
\partial_{\bar{z}} W(x, y)=\frac{i f^{\prime}(y)}{2 f(y)} \bar{W}(x, y)
$$

where

$$
f=\frac{c_{1}}{\sqrt{\alpha}} \sin \mathcal{A}+\frac{c_{2}}{\sqrt{\alpha}} \cos \mathcal{A} ;
$$

$\mathcal{A}$ is an antiderivative of $\alpha$ with respect to $y, c_{1}$ and $c_{2}$ are arbitrary real constants, $z=x+i y$ and $\partial_{\bar{z}}=\frac{1}{2}\left(\partial_{x}+i \partial_{y}\right)$. A complete (in a compact uniform convergence topology) system of exact solutions to (4) can be constructed 
explicitly. The system represents a set of formal powers [3], 6] which generalize the usual analytic complex powers $\left(z-z_{0}\right)^{n}, n=0,1,2, \ldots$ and in a sense give us a general solution of (44). Thus, in the case when $\alpha$ is a function of one Cartesian variable the Vekua equation equivalent to (2) in a two-dimensional situation can be solved and a complete system of solutions of (2) is obtained.

\section{Preliminaries}

We need the following definition. Consider the equation

$$
\partial_{\bar{z}} \varphi=\Phi
$$

on a whole complex plane or on a convex domain, where $\Phi=\Phi_{1}+i \Phi_{2}$ is a given complex valued function such that its real part $\Phi_{1}$ and imaginary part $\Phi_{2}$ satisfy the equation

$$
\partial_{y} \Phi_{1}-\partial_{x} \Phi_{2}=0
$$

then there exist real valued solutions of (5) which can be easily constructed in the following way

$$
\varphi(x, y)=2\left(\int_{x_{0}}^{x} \Phi_{1}(\eta, y) d \eta+\int_{y_{0}}^{y} \Phi_{2}\left(x_{0}, \xi\right) d \xi\right)+c
$$

where $\left(x_{0}, y_{0}\right)$ is an arbitrary fixed point in the domain of interest and $c$ is an arbitrary real constant.

By $\bar{A}$ we denote the integral operator in (7):

$$
\bar{A}[\Phi](x, y)=2\left(\int_{x_{0}}^{x} \Phi_{1}(\eta, y) d \eta+\int_{y_{0}}^{y} \Phi_{2}\left(x_{0}, \xi\right) d \xi\right)+c .
$$

Note that formula (7) can be extended to any simply connected domain by considering the integral along an arbitrary rectifiable curve $\Gamma$ leading from $\left(x_{0}, y_{0}\right)$ to $(x, y)$

$$
\varphi(x, y)=2\left(\int_{\Gamma} \Phi_{1} d x+\Phi_{2} d y\right)+c .
$$

Thus if $\Phi$ satisfies (6), there exists a family of real valued functions $\varphi$ such that $\partial_{\bar{z}} \varphi=\Phi$, given by the formula $\varphi=\bar{A}[\Phi]$. 
Let $f$ denote a given positive twice continuously differentiable function defined on a domain $\Omega \subset \mathbb{C}$. Consider the following Vekua equation

$$
W_{\bar{z}}=\frac{f_{\bar{z}}}{f} \bar{W} \quad \text { in } \Omega
$$

where the subindex $\bar{z}$ means the application of the operator $\partial_{\bar{z}}, W$ is a complex-valued function and $\bar{W}$ is its complex conjugate function. As was shown in [12], [13, [14, [15], 17] equation (8) is closely related to the secondorder equation of the form

$$
(\operatorname{div} p \operatorname{grad}+q) u=0 \quad \text { in } \Omega
$$

where $p$ and $q$ are real-valued functions. In particular the following statements are valid.

Theorem 1 [14] Let $u_{0}$ be a positive solution of (9). Assume that $f=\sqrt{p} u_{0}$ and $W$ is any solution of (8). Then $u=\frac{1}{\sqrt{p}} \operatorname{Re} W$ is a solution of (9)) and $v=\sqrt{p} \operatorname{Im} W$ is a solution of

$$
\left(\operatorname{div} \frac{1}{p} \operatorname{grad}+q_{1}\right) v=0 \quad \text { in } \Omega
$$

where

$$
q_{1}=-\frac{1}{p}\left(\frac{q}{p}+2\left\langle\frac{\nabla p}{p}, \frac{\nabla u_{0}}{u_{0}}\right\rangle+2\left(\frac{\nabla u_{0}}{u_{0}}\right)^{2}\right) .
$$

Theorem 2 [14] Let $\Omega$ be a simply connected domain, $u_{0}$ be a positive solution of (9) and $f=p^{1 / 2} u_{0}$. Assume that $u$ is a solution of (9)). Then a solution $v$ of (10) with $q_{1}$ defined by (11) such that $W=p^{1 / 2} u+i p^{-1 / 2} v$ is a solution of (8) is constructed according to the formula

$$
v=u_{0}^{-1} \bar{A}\left(i p u_{0}^{2} \partial_{\bar{z}}\left(u_{0}^{-1} u\right)\right)
$$

and vice versa, let $v$ be a solution of (10), then the corresponding solution $u$ of (9) such that $W=p^{1 / 2} u+i p^{-1 / 2} v$ is a solution of (8)), is constructed according to the formula

$$
u=-u_{0} \bar{A}\left(i p^{-1} u_{0}^{-2} \partial_{\bar{z}}\left(u_{0} v\right)\right) .
$$


Thus the relation between (8) and (9) is very similar to that between the Cauchy-Riemann system and the Laplace equation. Moreover, choosing $p \equiv 1, q \equiv 0$ and $u_{0} \equiv 1$ we obtain that (12) and (13) become the well known formulas from the classical complex analysis for constructing conjugate harmonic functions.

For a Vekua equation of the form

$$
W_{\bar{z}}=a W+b \bar{W}
$$

where $a$ and $b$ are arbitrary complex-valued functions from an appropriate function space [20] a well developed theory of Taylor and Laurent series in formal powers was created (see [3], 44) containing among others the expansion and the Runge theorems as well as more precise convergence results (see, e.g., [19]) and a general simple algorithm [15] for explicit construction of formal powers for the Vekua equation of the form (8)).

\section{Reduction of (2) to a Vekua equation}

We consider equation (2) where both $\alpha$ and $\vec{B}$ are supposed to be dependent on two Cartesian variables $x$ and $y$. Then equation (2) can be written as the following system

$$
\begin{gathered}
\partial_{y} B_{3}+\alpha B_{1}=0 \\
-\partial_{x} B_{3}+\alpha B_{2}=0 \\
\partial_{x} B_{2}-\partial_{y} B_{1}+\alpha B_{3}=0 .
\end{gathered}
$$

Solving this system for $B_{3}$ leads to the equation

$$
\Delta B_{3}-\left\langle\frac{\nabla \alpha}{\alpha}, \nabla B_{3}\right\rangle+\alpha^{2} B_{3}=0
$$

where $\langle\cdot, \cdot\rangle$ denotes the usual scalar product of two vectors.

Note that

$$
\alpha \operatorname{div}\left(\frac{1}{\alpha} \nabla B_{3}\right)=\Delta B_{3}-\left\langle\frac{\nabla \alpha}{\alpha}, \nabla B_{3}\right\rangle
$$

and hence (16) can be rewritten as follows

$$
\operatorname{div}\left(\frac{1}{\alpha} \nabla B_{3}\right)+\alpha B_{3}=0
$$


Thus equation (2) reduces to an equation of the form (9) with $p=1 / \alpha$ and $q=\alpha$.

Let us notice that (see, e.g., [14])

$$
\operatorname{div} \frac{1}{\alpha} \nabla+\alpha=\frac{1}{\sqrt{\alpha}}(\Delta-r) \frac{1}{\sqrt{\alpha}}
$$

where

$$
r=-\frac{1}{2} \frac{\Delta \alpha}{\alpha}+\frac{3}{4}\left(\frac{\nabla \alpha}{\alpha}\right)^{2}-\alpha^{2} .
$$

That is $B_{3}$ is a solution of (17) iff the function $f=B_{3} / \sqrt{\alpha}$ is a solution of the stationary Schrödinger equation

$$
(-\Delta+r) f=0
$$

with $r$ defined by (18). As was explained in section 2, given its particular solution this equation reduces to the Vekua equation (88). Unfortunately, in general we are not able to propose a particular solution of (17). Nevertheless in an important special case when $\alpha$ depends on one Cartesian variable, a particular solution of (17) is always available in explicit form. We give this result in the next section.

\section{Solution in the case when $\alpha$ is a function of one Cartesian variable}

Let us consider equation (19) where $\alpha=\alpha(y)$. We assume that $\alpha$ is a nonvanishing function and look for a solution of the corresponding ordinary differential equation

$$
\frac{d^{2} f_{0}}{d y^{2}}+\left(\frac{1}{2} \frac{\alpha^{\prime \prime}}{\alpha}-\frac{3}{4}\left(\frac{\alpha^{\prime}}{\alpha}\right)^{2}+\alpha^{2}\right) f_{0}=0
$$

Its general solution is known (see [10, $2.162(14)]$ ) and is given by the expression

$$
f_{0}(y)=\frac{c_{1}}{\sqrt{\alpha(y)}} \sin \mathcal{A}(y)+\frac{c_{2}}{\sqrt{\alpha(y)}} \cos \mathcal{A}(y)
$$

where $\mathcal{A}$ is an antiderivative of $\alpha$ and $c_{1}, c_{2}$ are arbitrary real constants. 
Choosing, e.g., $c_{1}=1, c_{2}=0$ and calculating the coefficient $\left(\partial_{\bar{z}} f_{0}\right) / f_{0}$ we arrive at the following Vekua equation which is equivalent to (2) in the case under consideration (and which is considered in any simply connected domain where $\sin \mathcal{A}(y)$ does not vanish),

$$
\partial_{\bar{z}} W(x, y)=\frac{i}{2}\left(\alpha(y) \cot \mathcal{A}(y)-\frac{\alpha^{\prime}(y)}{2 \alpha(y)}\right) \bar{W}(x, y) .
$$

Note that $F=f_{0}=\frac{\sin \mathcal{A}(y)}{\sqrt{\alpha(y)}}$ and $G=\frac{i}{f_{0}}=\frac{i \sqrt{\alpha(y)}}{\sin \mathcal{A}(y)}$ represent a generating pair for this Vekua equation (see [13], [15]) and hence if $W$ is its solution, the corresponding pseudoanalytic function of the second kind $\omega=\frac{1}{f_{0}} \operatorname{Re} W+$ $i f_{0} \operatorname{Im} W$ satisfies the equation

$$
\omega_{\bar{z}}=\frac{1-f_{0}^{2}}{1+f_{0}^{2}} \bar{\omega}_{\bar{z}}
$$

which can be written in the form of the following system

$$
\phi_{x}=\frac{1}{f_{0}^{2}} \psi_{y}, \quad \phi_{y}=-\frac{1}{f_{0}^{2}} \psi_{x}
$$

where $\phi=\operatorname{Re} \omega$ and $\psi=\operatorname{Im} \omega$.

For $f_{0}$ being representable in a separable form $f_{0}(x, y)=X(x) Y(y)$ the formulas for constructing corresponding formal powers explicitly were presented already by L. Bers and A. Gelbart (see [3] and [6]). Using them we obtain the following representation for the formal powers corresponding to (21)

$$
\begin{aligned}
{ }_{*} Z^{(n)}\left(a, z_{0} ; z\right) & =a_{1} \sum_{k=0}^{n}\left(\begin{array}{c}
n \\
k
\end{array}\right)\left(x-x_{0}\right)^{(n-k)} i^{k} Y^{k} \\
& +i a_{2} \sum_{k=0}^{n}\left(\begin{array}{c}
n \\
k
\end{array}\right)\left(x-x_{0}\right)^{(n-k)} i^{k} \widetilde{Y}^{k}
\end{aligned}
$$

(we preserve the notations from [3]) where $z_{0}=x_{0}+i y_{0}$ is an arbitrary point of the domain of interest, $a$ is an arbitrary complex number: $a=a_{1}+i a_{2}$, $Y^{k}$ and $\widetilde{Y}^{k}$ are constructed as follows

$$
Y^{(0)}\left(y_{0}, y\right)=\tilde{Y}^{(0)}\left(y_{0}, y\right)=1
$$


and for $n=1,2, \ldots$

$$
\begin{aligned}
Y^{(n)}\left(y_{0}, y\right) & =n \int_{y_{0}}^{y} Y^{(n-1)}\left(y_{0}, \eta\right) f_{0}^{2}(\eta) d \eta \quad n \text { odd } \\
Y^{(n)}\left(y_{0}, y\right) & =n \int_{y_{0}}^{y} Y^{(n-1)}\left(y_{0}, \eta\right) \frac{d \eta}{f_{0}^{2}(\eta)} \quad n \text { even } \\
\widetilde{Y}^{(n)}\left(x_{0}, x\right) & =n \int_{y_{0}}^{y} \widetilde{Y}^{(n-1)}\left(y_{0}, \eta\right) \frac{d \eta}{f_{0}^{2}(\eta)} \quad n \text { odd } \\
\widetilde{Y}^{(n)}\left(x_{0}, x\right) & =n \int_{y_{0}}^{y} \widetilde{Y}^{(n-1)}\left(y_{0}, \eta\right) f_{0}^{2}(\eta) d \eta \quad n \text { even. }
\end{aligned}
$$

The system $\left\{{ }_{*} Z^{(n)}\left(1, z_{0} ; z\right),{ }_{*} Z^{(n)}\left(i, z_{0} ; z\right)\right\}_{n=0}^{\infty}$ represents a complete (in a compact uniform convergence topology [2]) system of solutions of (21) that means that any solution $\omega$ of (21) in a simply connected domain $\Omega$ can be represented as a series

$$
\omega(z)=\sum_{n=0}^{\infty}{ }_{*} Z^{(n)}\left(a_{n}, z_{0} ; z\right)=\sum_{n=0}^{\infty}\left(a_{n *}^{\prime} Z^{(n)}\left(1, z_{0} ; z\right)+a_{n *}^{\prime \prime} Z^{(n)}\left(i, z_{0} ; z\right)\right)
$$

where $a_{n}^{\prime}=\operatorname{Re} a_{n}, a_{n}^{\prime \prime}=\operatorname{Im} a_{n}$ and the series converges normally (uniformly on any compact subset of $\Omega$ ). Consequently the system of functions

$$
\left\{f_{0}(y) \operatorname{Re}\left({ }_{*} Z^{(n)}\left(1, z_{0} ; z\right)\right), \quad f_{0}(y) \operatorname{Re}\left({ }_{*} Z^{(n)}\left(i, z_{0} ; z\right)\right)\right\}_{n=0}^{\infty}
$$

represents in the same sense a complete system of solutions of (19) with $r$ defined by (18), and

$$
\left\{\sqrt{\alpha(y)} f_{0}(y) \operatorname{Re}\left({ }_{*} Z^{(n)}\left(1, z_{0} ; z\right)\right), \quad \sqrt{\alpha(y)} f_{0}(y) \operatorname{Re}\left({ }_{*} Z^{(n)}\left(i, z_{0} ; z\right)\right)\right\}_{n=0}^{\infty}
$$

is a complete system of solutions of (17). Thus in the case under consideration any solution $B_{3}$ of (17) can be represented in the form

$B_{3}(x, y)=\sum_{n=0}^{\infty}\left(a_{n} \sin \mathcal{A}(y) \operatorname{Re}\left({ }_{*} Z^{(n)}\left(1, z_{0} ; z\right)\right)+b_{n} \sin \mathcal{A}(y) \operatorname{Re}\left({ }_{*} Z^{(n)}\left(i, z_{0} ; z\right)\right)\right)$ 
where $a_{n}$ and $b_{n}$ are real constants.

The other two components of the vector $\vec{B}$ are obtained from (14) and (15)):

$$
B_{1}=-\frac{1}{\alpha} \partial_{y} B_{3} \quad \text { and } \quad B_{2}=\frac{1}{\alpha} \partial_{x} B_{3}
$$

that gives us a complete system of solutions of (2) in the case under consideration. On the following example we explain how this procedure works.

Example 3 Let us consider the following relatively simple situation in which the corresponding integrals are not difficult to evaluate. Let

$$
\alpha(y)=\frac{1}{\sqrt{1-y^{2}}}
$$

and $\Omega$ be an open unitary disk with a center in the origin. We take in (20) $c_{1}=0$ and $c_{2}=1$. Then it is easy to verify that

$$
f_{0}(y)=\left(1-y^{2}\right)^{\frac{3}{4}}
$$

The first three formal powers with a centre in the origin can be calculated as follows

$$
\begin{gathered}
Z^{(1)}(1,0 ; z)=x+i\left[\frac{y\left(1-y^{2}\right)^{\frac{3}{2}}}{4}+\frac{3 y\left(1-y^{2}\right)^{\frac{1}{2}}}{8}+\frac{3}{8} \arcsin y\right] \\
* Z^{(1)}(i, 0 ; z)=-\frac{y}{\left(1-y^{2}\right)^{\frac{1}{2}}}+i x \\
{ }_{*} Z^{(2)}(1,0 ; z)=x^{2}-\frac{1}{4} y^{2}-\frac{3}{4} \frac{y \arcsin y}{\left(1-y^{2}\right)^{\frac{1}{2}}} \\
+2 i x\left(\frac{y\left(1-y^{2}\right)^{\frac{3}{2}}}{4}+\frac{3 y\left(1-y^{2}\right)^{\frac{1}{2}}}{8}+\frac{3}{8} \arcsin y\right) \\
* Z^{(2)}(i, 0 ; z)=-\frac{2 x y}{\left(1-y^{2}\right)^{\frac{1}{2}}}+i\left(x^{2}-y^{2}-\frac{1}{2} y^{4}\right)
\end{gathered}
$$




$$
\begin{aligned}
* Z^{(3)}(1,0 ; z) & =x^{3}-3 x\left(\frac{1}{4} y^{2}+\frac{3}{4} \frac{y \arcsin y}{\left(1-y^{2}\right)^{\frac{1}{2}}}\right) \\
& +3 i x^{2}\left(\frac{y\left(1-y^{2}\right)^{\frac{3}{2}}}{4}+\frac{3 y\left(1-y^{2}\right)^{\frac{1}{2}}}{8}+\frac{3}{8} \arcsin y\right) \\
& -i\left(-\frac{3}{24} y\left(1-y^{2}\right)^{\frac{5}{2}}+\frac{3}{96} y\left(1-y^{2}\right)^{\frac{3}{2}}+y\left(1-y^{2}\right)^{\frac{1}{2}}\left(\frac{51}{128}-\frac{9}{64} y^{2}\right)\right. \\
& \left.-\frac{9}{16}\left(1-y^{2}\right)^{2} \arcsin y+\frac{33}{128} \arcsin y\right), \\
* Z^{(3)}(i, 0 ; z)= & -\frac{3 x^{2} y}{\left(1-y^{2}\right)^{\frac{1}{2}}}+\frac{3}{4} \frac{y\left(1+y^{2}\right)}{\left(1-y^{2}\right)^{\frac{1}{2}}}-\frac{3}{4} \arcsin y+i x\left(x^{2}-3\left(y^{2}-\frac{1}{2} y^{4}\right)\right) .
\end{aligned}
$$

Now taking the real parts of these formal powers and multiplying them by the factor $\sqrt{\alpha} f_{0}$ (see (22)) we obtain the first elements of the complete system of solutions of (17), that is any solution $B_{3}$ of (17) in a simply connected domain can be represented as an infinite linear combination of the functions

$$
\begin{aligned}
& \left\{\left(1-y^{2}\right)^{\frac{1}{2}}, \quad x\left(1-y^{2}\right)^{\frac{1}{2}}, \quad-y, \quad\left(1-y^{2}\right)^{\frac{1}{2}}\left(x^{2}-\frac{1}{4} y^{2}-\frac{3}{4} \frac{y \arcsin y}{\left(1-y^{2}\right)^{\frac{1}{2}}}\right),\right. \\
& -2 x y, \quad\left(1-y^{2}\right)^{\frac{1}{2}}\left(x^{3}-3 x\left(\frac{1}{4} y^{2}+\frac{3}{4} \frac{y \arcsin y}{\left(1-y^{2}\right)^{\frac{1}{2}}}\right)\right), \\
& \left.-3 x^{2} y+\frac{3}{4} y\left(1+y^{2}\right)-\frac{3}{4}\left(1-y^{2}\right)^{\frac{1}{2}} \arcsin y, \ldots\right\}
\end{aligned}
$$

and the corresponding series converges normally.

From (23) it is easy to calculate the corresponding components $B_{1}$ and $B_{2}$ respectively,

$$
\begin{aligned}
& \left\{y, \quad x y, \quad\left(1-y^{2}\right)^{\frac{1}{2}}, \quad \frac{3}{4}\left(1-y^{2}\right)^{\frac{1}{2}} \arcsin y+y\left(x^{2}-\frac{3}{4} y^{2}+\frac{5}{4}\right),\right. \\
& 2 x\left(1-y^{2}\right)^{\frac{1}{2}}, \quad \frac{9}{4} x\left(1-y^{2}\right)^{\frac{1}{2}} \arcsin y+y\left(x^{3}-\frac{9}{4} x y^{2}+\frac{15}{4} x\right) \\
& \left.-\left(\frac{9}{4} y^{2}-3 x^{2}\right)\left(1-y^{2}\right)^{\frac{1}{2}}-\frac{3}{4} y \arcsin y, \ldots\right\}
\end{aligned}
$$

and

$$
\begin{aligned}
& \left\{0, \quad\left(1-y^{2}\right), \quad 0, \quad 2 x\left(1-y^{2}\right), \quad-2 y\left(1-y^{2}\right)^{\frac{1}{2}},\right. \\
& \left.\left(3 x^{2}-\frac{3}{4} y^{2}\right)\left(1-y^{2}\right)-\frac{9}{4} y\left(1-y^{2}\right)^{\frac{1}{2}} \arcsin y, \quad-6 x y\left(1-y^{2}\right)^{\frac{1}{2}}, \ldots\right\} \text {. }
\end{aligned}
$$


Thus, we obtain the following complete system of solutions of (2) with the proportionality factor $\alpha$ defined by (24),

$$
\begin{aligned}
& \vec{B}_{0}=\left(\begin{array}{c}
y \\
0 \\
\left(1-y^{2}\right)^{\frac{1}{2}}
\end{array}\right), \quad \vec{B}_{1}=\left(\begin{array}{c}
x y \\
\left(1-y^{2}\right) \\
x\left(1-y^{2}\right)^{\frac{1}{2}}
\end{array}\right), \quad \vec{B}_{2}=\left(\begin{array}{c}
\left(1-y^{2}\right)^{\frac{1}{2}} \\
0 \\
-y
\end{array}\right), \\
& \vec{B}_{3}=\left(\begin{array}{c}
\frac{3}{4}\left(1-y^{2}\right)^{\frac{1}{2}} \arcsin y+y\left(x^{2}-\frac{3}{4} y^{2}+\frac{5}{4}\right) \\
2 x\left(1-y^{2}\right) \\
\left(1-y^{2}\right)^{\frac{1}{2}}\left(x^{2}-\frac{1}{4} y^{2}-\frac{3}{4} \frac{y \arcsin y}{\left(1-y^{2}\right)^{\frac{1}{2}}}\right)
\end{array}\right), \quad \vec{B}_{4}=\left(\begin{array}{c}
2 x\left(1-y^{2}\right)^{\frac{1}{2}} \\
-2 y\left(1-y^{2}\right)^{\frac{1}{2}} \\
-2 x y
\end{array}\right), \\
& \vec{B}_{5}=\left(\begin{array}{c}
\frac{9}{4} x\left(1-y^{2}\right)^{\frac{1}{2}} \arcsin y+y\left(x^{3}-\frac{9}{4} x y^{2}+\frac{15}{4} x\right) \\
\left(3 x^{2}-\frac{3}{4} y^{2}\right)\left(1-y^{2}\right)-\frac{9}{4} y\left(1-y^{2}\right)^{\frac{1}{2}} \arcsin y \\
\left(1-y^{2}\right)^{\frac{1}{2}}\left(x^{3}-3 x\left(\frac{1}{4} y^{2}+\frac{3}{4} \frac{y \arcsin y}{\left(1-y^{2}\right)^{\frac{1}{2}}}\right)\right)
\end{array}\right) \\
& \vec{B}_{6}=\left(\begin{array}{c}
-\left(\frac{9}{4} y^{2}-3 x^{2}\right)\left(1-y^{2}\right)^{\frac{1}{2}}-\frac{3}{4} y \arcsin y \\
-6 x y\left(1-y^{2}\right)^{\frac{1}{2}} \\
-3 x^{2} y+\frac{3}{4} y\left(1+y^{2}\right)-\frac{3}{4}\left(1-y^{2}\right)^{\frac{1}{2}} \arcsin y
\end{array}\right)
\end{aligned}
$$

\section{Concluding remarks}

We presented two new results.

1. We showed that the system of equations describing Beltrami fields on the plane can be reduced to a Vekua equation of the form (8) whenever any particular solution of the corresponding second-order equation of the form (17) or (19) is known.

2 . In the case when the proportionality factor $\alpha$ depends on one Cartesian variable we obtain a particular solution explicitly, construct the corresponding Vekua equation (section 4), and solve it in the sense that a complete system of solutions is obtained which gives us a complete system of solutions of the original vector equation describing Beltrami fields. 
Of course not always the integrals involved in the construction of the complete system of solutions are sufficiently easy to evaluate explicitly as in the example 3. Nevertheless our numerical experiments confirm that in general the formal powers and hence the solutions of (2) can be calculated with a remarkable accuracy. For example, the vector $\vec{B}_{40}$ (see notations in the example 3) in the Matlab 7 package on a usual PC can be calculated with a precision of the order $10^{-4}$. Thus, the use of formal powers for numerical solution of boundary value problems corresponding to (2) and more generally to equations of the form (9) is really promising. The work in this direction will be reported elsewhere.

\section{References}

[1] Athanasiadis C, Costakis G and Stratis I G 2000 On some properties of Beltrami fields in chiral media. Reports on Mathematical Physics 45 $257-271$.

[2] Bers L 1950 The expansion theorem for sigma-monogenic functions. American Journal of Mathematics 72 705-712.

[3] Bers L 1952 Theory of pseudo-analytic functions. New York University.

[4] Bers L 1956 Formal powers and power series. Communications on Pure and Applied Mathematics 9 693-711.

[5] Castañeda A and Kravchenko V V 2005 New applications of pseudoanalytic function theory to the Dirac equation. J. of Physics A: Mathematical and General 38, No. 42 9207-9219.

[6] Courant R and Hilbert D 1989 Methods of Mathematical Physics, v. 2. Wiley-Interscience.

[7] Feng Qingzeng 1997 On force-free magnetic fields and Beltrami flows. Applied Mathematics and Mechanics (English Edition) 18 997-1003.

[8] Gonzalez-Gascon F and Peralta-Salas D 2001 Ordered behaviour in force-free magnetic fields. Physics Letters A 292 75-84. 
[9] Kaiser R, Neudert M and von Wahl W 2000 On the existence of force-free magnetic fields with small nonconstant $\alpha$ in exterior domains. Communications in Mathematical Physics 211 111-136.

[10] Kamke E 1976 Handbook of ordinary differential equations. Moscow: Nauka (Russian translation from the German original: 1959 Differentialgleichungen. Lösungsmethoden und Lösungen. Leipzig).

[11] Kravchenko V V 2003 On Beltrami fields with nonconstant proportionality factor. J. of Phys. A 36, 1515-1522.

[12] Kravchenko V V 2005 On the reduction of the multidimensional stationary Schrödinger equation to a first order equation and its relation to the pseudoanalytic function theory. J. of Phys. A 38, No. 4, 851-868.

[13] Kravchenko V V 2005 On a relation of pseudoanalytic function theory to the two-dimensional stationary Schrödinger equation and Taylor series in formal powers for its solutions. J. of Phys. A , 38, No. 18, 3947-3964.

[14] Kravchenko V V 2006 On a factorization of second order elliptic operators and applications. Journal of Physics A: Mathematical and General 39, No. 40, 12407-12425.

[15] Kravchenko V V Recent developments in applied pseudoanalytic function theory. To appear, available from www.arxiv.org.

[16] Kravchenko V V and Oviedo H 2003 On a quaternionic reformulation of Maxwell's equations for chiral media and its applications. Zeitschrift für Analysis und ihre Anwendungen 22, No. 3, 569 - 589.

[17] Kravchenko V V and Oviedo H 2007 On explicitly solvable Vekua equations and explicit solution of the stationary Schrödinger equation and of the equation $\operatorname{div}(\sigma \nabla u)=0$. Complex Variables and Elliptic Equations 52, No. 5, 353 - 366.

[18] Lakhtakia A 1994 Beltrami fields in chiral media. Singapore: World Scientific.

[19] Menke K 1974 Zur Approximation pseudoanalytischer Funktionen durch Pseudopolynome. Manuscripta Math. 11 111-125. 
[20] Vekua I N 1959 Generalized analytic functions. Moscow: Nauka (in Russian); English translation Oxford: Pergamon Press 1962.

[21] Yoshida Z 1997 Applications of Beltrami functions in plasma physics. Nonlinear Analysis, Theory \& Applications 30 3617-3627.

[22] Zaghloul H and Barajas O 1990 Force-free magnetic fields. American Journal of Physics 58 (8) 783-788. 